\title{
Composite MET-PET Fusion 3.0 Tesla MRI is a New Sensitive Method for the Detection of GH Secreting Adenomas with Abnormal GH Secretary Dynamics
}

\section{Shunsuke Satoh ${ }^{1}$, Hidetoshi Ikeda ${ }^{1 *}$, Toshiyuki Saginoya ${ }^{2}$ and Kazuo Watanabe ${ }^{3}$}

${ }^{1}$ Reseach Institute for Pituitary Disease, Southern Tohoku General Hospital, Koriyama, Fukushima 963-8563, Japan

${ }^{2}$ Department of Radiology, Southern Tohoku General Hospital, Koriyama, Fukushima 963-8563, Japan

${ }^{3}$ Reseach Institute for Neuroscience, Southern Tohoku General Hospital, Koriyama, Fukushima 963-8563, Japan

\begin{abstract}
Objective: To evaluate the usefulness of methionine (MET)-Positron Emission Tomography (PET) for the early diagnosis of Growth Hormone $(\mathrm{GH})$ secreting adenomas, we retrospectively analyzed patients with abnormal GH dynamics and a histologically confirmed GH secreting pituitary adenoma who underwent pre-operative fluorodeoxyglucose-/MET-PET.

Materials and methods: Thirty-two patients with abnormal GH dynamics were included in this study. 3 Tesla (3T) -MRI, $\left[{ }^{18} \mathrm{~F}\right]$-flurodeoxyglucose (FDG) $-/\left[{ }^{11} \mathrm{C}\right]-\mathrm{MET}-\mathrm{PET}$, and transsphenoidal surgery were performed in all the patients. We determined correlations among FDG-/MET-PET (maximum standardized uptake value (SUVmax)), serum GH and IGF-1 levels, the GH response to a $75 \mathrm{~g}$ Oral Glucose Tolerance Test (OGTT), Thyrotropin-Releasing Hormone (TRH) /Luteinizing Hormone-Releasing Hormone (LHRH) loading tests, tumor volume, and proliferative activity.

Results: The diagnostic sensitivity for early GH secreting adenoma was highest for MET-PET (100\%), followed by TRH/LHRH (80\%), $75 \mathrm{~g}$ OGTT (67\%), and FDG-PET (47\%). Significant correlations were observed between: MET-PET SUVmax and FDG-PET SUVmax $(p<0.01)$, MET-/FDG-PET SUVmax and tumor volume (MET: $p<0.01$; FDG: $p<0.01$ ) ; MET-/FDG PET SUVmax and MIB-1 labeling index (MET: $p<0.01$; FDG: $p=0.01$ ) ; GH/insulin-like growth factor (IGF)-1 levels and tumor volume (GH: $p=0.01$; IGF-1: $p<0.01)$; and GH and IGF-1 levels $(p<0.01)$.
\end{abstract}

Conclusions: All the patients with abnormal GH dynamics detected by MET-PET had GH adenomas, indicating that MET-PET is useful for the early diagnosis of $\mathrm{GH}$ adenomas.

Keywords: Positron emission tomography; Methionine; Acromegaly; Magnetic resonance imaging; Fluorodeoxyglucose

\section{Introduction}

Sustained Growth Hormone (GH) excess resulting from a GH secreting pituitary adenoma causes acromegaly, the diagnosis of which ranges from 2.5 to 10 years (mean 8 years) after the onset of symptoms [1-6]. Acromegaly is associated with a 2-3-fold higher mortality rate and a reduction of life expectancy by $10-15$ years compared with the general population. These outcomes are mainly attributed to the presence of other severe diseases, such as diabetes mellitus, hypertension, hyperlipidemia, cardiovascular and respiratory disease, and neoplasms [1,3,5,7-13]. The duration of the disease is a prognostic factor. Therefore, establishing guidelines for the early diagnosis or assessment of the disease activity of acromegaly is a major challenge $[1,4,6,10]$. Acromegaly, which sometimes shows a false-negative or false-positive reaction to various hormone-loading tests, does not always fulfill the established diagnostic criteria. Moreover, neuroradiological assessments, such as computed tomography (CT) or Magnetic Resonance Imaging (MRI), sometimes fail to detect GH secreting adenomas. Recent reports have demonstrated the usefulness of Positron Emission Tomography (PET) for identifying pituitary adenomas [14-18]. In fact, methionine (MET)PET was able to detect tumor extension when it could not be detected with conventional imaging $[16,18]$. In the present study, we reviewed patients with abnormal GH secretion and a GH secreting adenoma that was histologically confirmed using a surgical specimen. We analyzed correlations among Fluorodeoxyglucose (FDG) -/MET-PET (maximum Standardized Uptake Value (SUVmax)), serum GH and IGF-1 levels, the GH response to a $75 \mathrm{~g}$ OGTT, TRH/LHRH loading tests, tumor volume, and proliferative activity. We also sought to identify the factors that facilitated the early diagnosis of GH secreting adenoma.

\section{Methods}

\section{Patients}

We identified 32 patients who fulfilled at least one of the following inclusion criteria: (1) high GH and/or IGF-1 levels; (2) no suppression of GH levels during a $75 \mathrm{~g}$ Oral Glucose Tolerance Test (OGTT) ; and (3) abnormal GH responses to Thyrotropin-Releasing Hormone (TRH) /luteinizing hormone-releasing hormone (LHRH) loading tests. Nine patients were male and 23 were female. The mean age was 44.3 years (range 15-68 years). Of the 32 patients with abnormal GH secretary dynamics, 17 had "overt" acromegaly. The other 15 patients had no apparent clinical characteristics of acromegaly, corresponding to pre-clinical acromegaly. All patients underwent neuroradiologic examinations (MRI and FDG-/MET-PET) and transsphenoidal surgery.

We analyzed the correlations among the following parameters: FDG-/ MET-PET (SUVmax values), GH and IGF-1 levels, the GH response

*Corresponding author: Hidetoshi lkeda, Research Institute for Pituitary Disease Southern Tohoku Research Institute for Neuroscience, 7-115 Yatsuyamada, Koriyama, Fukushima 963-8563, Japan, Tel: +81-24-934-5322; Fax: +81-24-922 5320; E-mail: ikeda@nsg.med.tohoku.ac.jp

Received September 21, 2012; Accepted November 25, 2012; Published November 29, 2012

Citation: Satoh S, Ikeda H, Saginoya T, Watanabe K (2012) Composite MET-PET Fusion 3.0 Tesla MRI is a New Sensitive Method for the Detection of GH Secreting Adenomas with Abnormal GH Secretary Dynamics. J Metabolic Synd S2:005. doi:10.4172/2167-0943.S2-005

Copyright: ( 2012 Satoh S, et al. This is an open-access article distributed under the terms of the Creative Commons Attribution License, which permits unrestricted use, distribution, and reproduction in any medium, provided the original author and source are credited. 
Citation: Satoh S, Ikeda H, Saginoya T, Watanabe K (2012) Composite MET-PET Fusion 3.0 Tesla MRI is a New Sensitive Method for the Detection of GH Secreting Adenomas with Abnormal GH Secretary Dynamics. J Metabolic Synd S2:005. doi:10.4172/2167-0943.S2-005

Page 2 of 5

to a $75 \mathrm{~g}$ OGTT, the GH response to TRH/LHRH loading tests, tumor volume, and proliferative activity. The objective of these analyses was to identify parameters that may facilitate early diagnosis. In patients whose GH and IGF-1 levels were measured several times, we calculated the mean values and determined their correlations with the other parameters. The magnitude of the GH fluctuation before surgery was compared between patients with acromegaly and pre-clinical acromegaly. FDG-/MET-PET scans were performed in 33 patients without pituitary disorders as a control. This study was conducted in accordance with the declaration of Helsinki. All the subjects provided informed consent.

\section{MRI}

MRI was performed using a 3 Tesla (3T) -MRI unit (Signa HDx; GE Medical Systems, Fairfield, CT, USA). A standard circular-polarized head coil was used. MRI of the brain was performed in the sagittal, coronal, and axial planes, and consisted of T1-weighted spin-echo images (TR/TE/number of excitations: $575 \mathrm{~ms} / 10 \mathrm{~ms} / 2$; matrix size: $256 \times 160)$ and T2-weighted spin-echo images $(\mathrm{TR} / \mathrm{TE} /$ number of excitations: $4400 \mathrm{~ms} / 102 \mathrm{~ms} / 2$; matrix size: $480 \times 192$ ). The slice thickness was $2 \mathrm{~mm}$, with a $0.2-\mathrm{mm}$ slice gap. For contrast-enhanced studies, $0.2 \mathrm{ml} / \mathrm{kg}$ body weight of gadolinium-diethylenetriamine pentaacetic acid was injected intravenously.

\section{PET imaging}

PET-CT was performed using a Discovery LS (GE Medical Systems) in all the patients. The PET machine used bismuth germanium oxide crystals. All the patients fasted before the procedure and received intravenous injections of MET $(5.6 \mathrm{MBq} \times$ body weight (dose range 225.7-558.3 MBq; 6.1-15.1 mCi)). PET scans, with duration of $10 \mathrm{~min}$, were obtained starting $20 \mathrm{~min}$ after injection. At $1 \mathrm{~h}$ after MET injection, all patients received an intravenous injection of FDG (3.7 MBq $\times$ body weight (dose range 155-269.5 MBq, i. e. 4.2-7.3 mCi)). PET scans, with duration of $10 \mathrm{~min}$, were obtained starting $1 \mathrm{~h}$ after injection. The FDG-/MET-PET procedure was performed in the three-dimensional mode, which provided a set of 35 planes with a section thickness of $\sim 4.1$ $\mathrm{mm}$. The uptake of FDG and MET during PET scanning was evaluated as the SUVmax.

\section{Image post-processing}

The MRI and PET scans were co-registered to Gd-enhanced T1weighted images and T2-weighted images using Advantage Windows software (GE Medical Systems). For co-registration of MRI and PET scans, the MRI data were reconstructed using the skull shape ascertained by PET-CT. This procedure was performed based on anatomical landmarks, such as the optic nerves, the internal occipital protuberance, and the vestibular cochlear nerve. The MRI scans were then fused to the PET scan; the overlaid PET scans were then automatically viewed.

\section{Pathologic examination}

Surgical specimens were fixed in $10 \%$ neutral-buffered formalin and

\begin{tabular}{|c|c|c|c|c|c|c|c|c|c|c|}
\hline Case No & Age(Yrs) Sex & Pathology & MET (SUVmax) & FDG SUVmax) & Volume(CM3) Size & MIB-1 (/HP) & $\mathrm{GH}$ & IGF-1 & 75g-OGTT & TRH/LHRH \\
\hline 1 & $68, F$ & Acro & 2.3 & 0 & 0.3 ,macro & 1.5 & 5.76 & 553 & - & $\mathrm{NE}$ \\
\hline 2 & $28, F$ & Acro & 8.3 & 20.2 & 4.03, macro & 3.4 & 23.6 & 1390 & - & NE \\
\hline 3 & $49, \mathrm{~F}$ & Acro & 6.2 & 10.4 & 16.1, macro & 2 & 5.77 & 824 & - & NE \\
\hline 4 & $61, F$ & Acro & 2.65 & 4.64 & 0.84 , macro & 2.7 & 13.8 & 702 & - & NE \\
\hline 5 & $54, \mathrm{M}$ & Acro & 3.8 & 6.1 & 4.33, macro & 1.7 & 11 & 1330 & - & + \\
\hline 6 & $23, F$ & Pre Arco & 2.8 & 0 & 0.32, micro & 1.7 & 4.88 & 393 & + & + \\
\hline 7 & $61, \mathrm{M}$ & Acro & 2.3 & 2 & 0.63 , macro & 2 & 22.02 & 508 & - & + \\
\hline 8 & $65, M$ & Acro & 4.9 & 6.5 & 2.62 , macro & 5.4 & 77 & 488 & - & NE \\
\hline 9 & $47, \mathrm{~F}$ & Pre Arco & 2.5 & 0 & 0.27, micro & 0.2 & 7.46 & 129.3 & + & - \\
\hline 10 & $60, \mathrm{M}$ & Acro & 1.7 & 0 & 0.18, micro & 1.1 & 9.05 & 130 & + & $\mathrm{NE}$ \\
\hline 11 & $21, \mathrm{M}$ & Pre Arco & 1.6 & 0 & 0.21, micro & 3.1 & 4.02 & 203 & - & + \\
\hline 12 & $59, \mathrm{~F}$ & Acro & 2.9 & 6.6 & 0.31 , micro & 6.6 & 2 & 472 & - & + \\
\hline 13 & $62, F$ & Pre Arco & 3.2 & 7.9 & 0.76 ,macro & 2 & 1.09 & 255 & + & - \\
\hline 14 & $31, F$ & Acro & 9.5 & 32.5 & 1 ,macro & 7.9 & 19.2 & 645.5 & - & + \\
\hline 15 & $58, F$ & Acro & 3.4 & 6.3 & 0.82 , macro & 1.9 & 7.7 & 976 & - & + \\
\hline 16 & $30, F$ & Pre Arco & 4 & 0 & 0.2 , macro & 3.5 & 0.85 & 226 & - & + \\
\hline 17 & $44, F$ & Pre Arco & 2.8 & 0 & 0.1 , micro & 1.4 & 0.43 & 199.5 & + & + \\
\hline 18 & $33, F$ & Pre Arco & 6.6 & 0 & 0.14, micro & 7.1 & 9.3 & 187 & & - \\
\hline 19 & $57, F$ & & 4.1 & 4.6 & 2.1, macro & 2 & 38.3 & 754 & $\mathrm{NE}$ & $\mathrm{NE}$ \\
\hline 20 & $33, F$ & Pre Arco & 2.4 & 0 & 0.05, micro & 0.2 & 5.83 & 174.7 & - & + \\
\hline 21 & $35, F$ & Pre Arco & 9.8 & 11.1 & 2.43 , macro & 2.2 & 5.88 & 507.3 & - & + \\
\hline 22 & $15, F$ & Pre Arco & 2.5 & 3.5 & 0.018, micro & 0 & 3.4 & 369.3 & - & + \\
\hline 23 & $17, \mathrm{M}$ & Pre Arco & 2.1 & 4 & 0.006 , micro & 0.72 & 0.7 & 397.2 & - & + \\
\hline 24 & $68, F$ & Pre Arco & 3.8 & 5 & 0.25 , macro & 0.5 & 3.37 & 161 & - & + \\
\hline 25 & $34, F$ & Pre Arco & 4 & 3 & 0.13 , macro & 0.53 & 7.68 & 228 & + & + \\
\hline 26 & $53, F$ & Acro & 4.6 & 12.9 & 0.35 , macro & 1.84 & 5.67 & 438 & - & + \\
\hline 27 & $59, F$ & Acro & 4.5 & 4.1 & 0.21 , macro & 8.94 & 0.07 & 106.7 & + & + \\
\hline 28 & $42, \mathrm{M}$ & Acro & 2.8 & 4.5 & 0.29 , macro & 3.3 & 7.22 & 763 & - & + \\
\hline 29 & $16, \mathrm{M}$ & Pre Arco & 3.6 & 3.3 & 0.2, micro & 1.6 & 5.11 & 291.7 & - & + \\
\hline 30 & $51, \mathrm{~F}$ & Acro & 2 & 4.1 & 1.68,macro & 0.2 & 9.87 & 792 & - & + \\
\hline 31 & $61, \mathrm{~F}$ & Acro & 3.6 & 7 & 4.32, macro & 1 & 23.4 & 467 & - & $\mathrm{NE}$ \\
\hline 32 & $25, F$ & Pre Arco & 2.3 & 0 & 0.14, micro & 1.5 & 2.65 & 276.5 & - & + \\
\hline
\end{tabular}

Table 1: Patient characteristics

Acro: acromegaly; preAcro: preclinical acromegaly; macro: macroadenoma; micro: microadenoma; $75 \mathrm{~g}$ OGTT: $+=$ suppression, $-=$ no suppression; TRH/LHRH: $+=$ paradoxical response; NE: not examined. 
Citation: Satoh S, Ikeda H, Saginoya T, Watanabe K (2012) Composite MET-PET Fusion 3.0 Tesla MRI is a New Sensitive Method for the Detection of GH Secreting Adenomas with Abnormal GH Secretary Dynamics. J Metabolic Synd S2:005. doi:10.4172/2167-0943.S2-005

embedded in paraffin. Tissue sections $(3 \mu \mathrm{m}$ thick) were prepared and stained with hematoxylin and eosin. Immunohistochemical staining was performed using the avidin-biotin complex method with the following antibodies: polyclonal adrenocorticotropic hormone (DAKO, Glostrup, Denmark), polyclonal growth hormone (DAKO), polyclonal prolactin (DAKO), monoclonal thyroid-stimulating hormone- $\beta$ (Neo Markers, Fremont, CA, USA), monoclonal luteinizing hormone- $\beta$ (Neo Markers), monoclonal follicle-stimulating hormone- $\beta$ (Neo Markers), polyclonal $\alpha$-subunit (DAKO), and monoclonal Ki-67 (DAKO). Tumor proliferative activity was evaluated in terms of the MIB-1 labeling index, which was calculated as the percentage of MIB-1-positive cells in a population of approximately 1000 tumor cells.

\section{Statistical analysis}

Since none of the parameters showed a normal distribution, Spearman's rank correlation test was used. Values of $\mathrm{p}<0.05$ were considered statistically significant. To compare the differences between two independent groups, where each group showed a normal distribution, Welch's $t$-test was used.

\section{Results}

The clinical data for each patient are summarized in table 1. High FDG accumulation was observed in $22 / 32$ patients (69\%), but was not observed in $4 / 13$ patients (31\%) with microadenoma. In contrast, high MET accumulation was observed in all 32 patients (Figure 1). In patients with microadenoma, we could delineate the tumor based on the difference in MET uptake between normal pituitary gland tissue and adenoma tissue. MET-PET SUVmax was significantly correlated with FDG-PET SUVmax $(p<0.01)$. Tumor volume was significantly correlated with MET-PET SUVmax $(\mathrm{p}<0.01)$ and FDG-PET SUVmax $(\mathrm{p}<0.01)$. The lesions detected by MET-PET fusion MRI, based on the high tracer uptake, were confirmed by the operative findings in all 32 patients, illustrating a high level of accuracy. We also found significant correlations between MET-PET SUVmax and MIB-1 ( $<<0.01$ ), FDGPET SUVmax and MIB-1 $(\mathrm{p}<0.01)$, FDG-PET SUVmax and IGF-1 levels $(\mathrm{p}<0.01), \mathrm{GH}$ levels and tumor volume $(\mathrm{p}=0.01)$, IGF-1 levels and tumor volume $(\mathrm{p}<0.01)$, and GH and IGF-1 levels $(\mathrm{p}<0.01)$ (Table 2$)$.

Microadenomas were detected in 11/15 patients with pre-clinical acromegaly (73\%) (Table 1). GH levels determined during the 75 g OGTT were not suppressed in 10/15 patients (67\%). Meanwhile, abnormal GH responses to the TRH/LHRH loading tests were observed in $12 / 15$ patients (80\%). High MET accumulation was observed in all 15 patients, whereas high FDG accumulation was only observed in 7/15 patients (47\%), including $4 / 4$ patients with macroadenoma (100\%) and $3 / 11$ patients with microadenoma (27\%). These findings suggest that the most sensitive diagnostic method for pre-clinical acromegaly is MET-PET, followed by TRH/LHRH, $75 \mathrm{~g}$ OGTT, and FDG-PET.

In the 33 control cases, the mean \pm standard deviation MET uptake was $1.7 \pm 0.47$ while that of FDG was $3.1 \pm 0.56$. The fluctuations in GH levels in patients with pre-clinical acromegaly and acromegaly are shown in figure 2. The fluctuations in GH levels were greater in patients with pre-clinical acromegaly as compared to patients with acromegaly ( $\mathrm{p}=0.03$, Welch's $t$-test $)$.

\section{Discussion}

It is important to diagnose GH secreting adenoma as early as possible to improve the prognosis, as prompt treatment can stop the progression of organ failure. Additionally, the surgical remission rate is high in patients with microadenoma $[1,3,4,11,19,20]$. For the diagnosis of acromegaly, it is important to measure the GH response to the 75

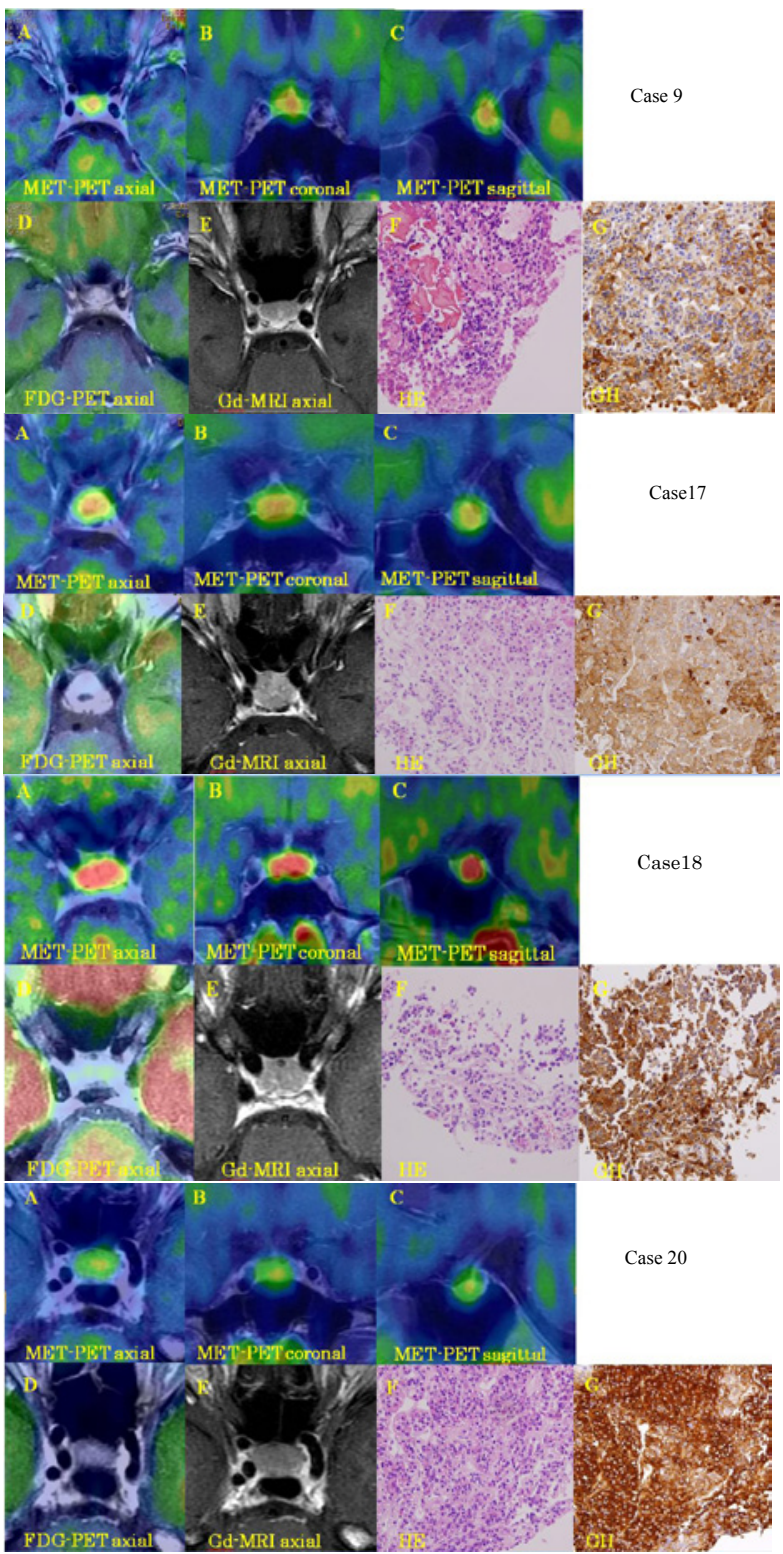

Figure 1: MET-PET fusion 3T MRI (A-C), FDG-PET/MRI (D), Gd-enhanced MRI (E), hematoxylin/eosin-stained tissue $(F)$, and immunohistochemical staining of $\mathrm{GH}(\mathrm{G})$ in four representative patients.

Patient \#9: high GH levels, GH suppression during a $75 \mathrm{~g}$ OGTT, normal $\mathrm{GH}$ responses to $\mathrm{TRH} / \mathrm{LHRH}$ loading tests, and MET-SUVmax $=2.5$.

Patient \#17: normal GH levels, GH suppression during a $75 \mathrm{~g}$ OGTT, abnormal $\mathrm{GH}$ responses to TRH/LHRH loading tests, and MET-SUVmax $=2.8$.

Patient \#18: high GH levels, no $\mathrm{GH}$ suppression during a $75 \mathrm{~g}$ OGTT, normal $\mathrm{GH}$ responses to TRH/LHRH loading tests, and MET-SUVmax = 6.6 .

Patient \#20: high GH levels, no GH suppression during a $75 \mathrm{~g}$ OGTT suppression, abnormal $\mathrm{GH}$ responses to TRH/LHRH loading tests, and MET-SUVmax $=2.4$

g OGTT suppression test. However, it was recently reported that the traditional methods used to measure GH have a high cutoff value, which may result in false-negative diagnosis [1-3,6,21-23]. Freda [24] concluded that a cutoff value of $1 \mu \mathrm{g} / \mathrm{l}$ fails to identify $50 \%$ of patients with active disease. Moreover, we must consider the influence of GH and IGF-1 levels in other conditions, such as diabetes mellitus, hepatic 


\begin{tabular}{|c|c|c|c|c|c|c|}
\hline & MET & FDG & MIB-1 & GH & IGF-1 & volume \\
\hline MET & & $\mathrm{P}<0.01$ & $\mathrm{P}<0.01$ & $\mathrm{P}=0.19$ & $\mathrm{P}=0.24$ & $\mathrm{P}<0.01$ \\
\hline FDG & $\mathrm{P}<0.01$ & & $\mathrm{P}=0.01$ & $\mathrm{P}=0.26$ & $\mathrm{P}<0.01$ & $\mathrm{P}<0.01$ \\
\hline MIB-1 & $\mathrm{P}<0.01$ & $\mathrm{P}=0.01$ & & $\mathrm{P}=0.52$ & $\mathrm{P}=0.20$ & $\mathrm{P}=0.12$ \\
\hline GH & $\mathrm{P}=0.19$ & $\mathrm{P}=0.26$ & $\mathrm{P}=0.52$ & & $\mathrm{P}<0.01$ & $\mathrm{P}<0.01$ \\
\hline IGF-1 & $\mathrm{P}=0.24$ & $\mathrm{P}<0.01$ & $\mathrm{P}=0.20$ & $\mathrm{P}<0.01$ & & $\mathrm{P}<0.01$ \\
\hline volume & $\mathrm{P}<0.01$ & $\mathrm{P}<0.01$ & $\mathrm{P}=0.12$ & $\mathrm{P}<0.01$ & $\mathrm{P}<0.01$ & \\
\hline
\end{tabular}

Table 2: Correlations between the indicated parameters.
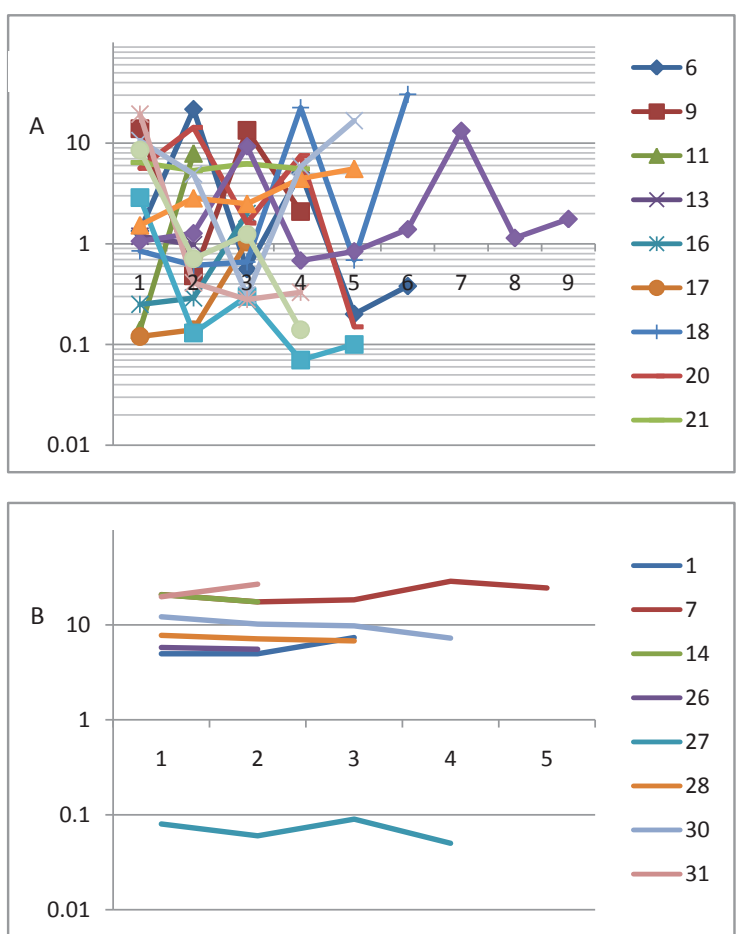

Figure 2: Fluctuations in plasma $\mathrm{GH}$ levels in 15 patients with pre-clinical acromegaly $(A)$ and in eight patients with overt acromegaly $(B)$.

or renal failure, hypothyroidism, pregnancy, adolescence, and anorexia nervosa, and that they are influenced by oral estrogen, age and sex [3,8,21,23-25]. About $50 \%$ of patients with acromegaly experience a rapid increase in plasma $\mathrm{GH}$ concentrations after stimulation of the TRH secretion and/or gonadotropin-releasing hormone $(\mathrm{GnRH})$ secretion. However, this paradoxical GH response to TRH/LHRH also occurs in conditions not associated with acromegaly such as depression, psychosis, liver cirrhosis, chronic renal failure, and uncontrolled type 1 diabetes mellitus, and may also occur in healthy women or adolescents. Therefore, the results of TRH/GnRH loading tests should provide part of the reference data sets $[3,26]$. Some reports attempted to elucidate tumor localization and the hormone secreting type from neuroradiological findings, but the results were inconclusive [22,27,28]. For the detection and delineation of tumor location, particularly of microadenomas, the diagnostic accuracy of superconductive MRI was reported to range from $10 \%$ to $40 \%$, with false-negative and falsepositive rates of $33 \%$ and $20 \%$, respectively $[16,18,29]$. Dynamic MRI is considered to improve diagnostic accuracy relative to superconductive MRI. However, Tabarin et al. [30] determined the diagnostic accuracy of MRI and dynamic MRI using 1.0 Tesla MRI. In that study, the positive rates were $53 \%$ for MRI and $67 \%$ for dynamic MRI, and were not significantly different between the two imaging modalities.

Pituitary tumor cells have a large capacity for synthesizing immature protein, irrespective of the type of hormone secreted, and a higher cell density than that found in normal pituitary tissue. MET is an important component for protein synthesis. Consequently, there is a marked difference in MET uptake between adenoma tissue and normal pituitary tissue, which contributes to the detection of tumors, including microadenomas $[16,18]$. In the present study, high MET uptake was observed in all 32 patients. Patients with a MET SUVmax $<2.0$ tend to have a microadenoma. In these patients, the difference in MET uptake between healthy tissue and adenoma tissue enabled us to delineate the tumor from the normal pituitary gland. In this study, the proportion of adenomas detected using FDG-PET was 69\%, which is lower than that achieved using MET-PET (100\%). We suspect that, in GH secreting adenoma, the uptake and metabolism of glucose and MET are facilitated by tumor volume and tumor proliferative activity. Consequently, both of these parameters were correlated with FDG/MET uptake. Furthermore, the SUVmax values for glucose and MET uptake were significantly correlated. Thus, MET-PET is an important factor to aid early diagnosis of microadenoma. In our study, the variation in $\mathrm{GH}$ levels was much higher in patients with pre-clinical acromegaly and microadenoma than in patients with acromegaly (Figure 2). However, the GH suppression after the $75 \mathrm{~g}$ OGTT loading test and the GH response to the TRH/ LHRH loading tests were inconclusive. Considering the correlations between GH/IGF-1 levels and tumor volume, hormone secretion tended to be much lower in patients with microadenoma compared to patients with macroadenoma. GH secretion shows a pulsatile pattern in normal individuals and in patients with acromegaly. Particularly in the early stages of acromegaly, the endocrine findings vary considerably, and assessment of GH levels is often unreliable (Figure 2). Considering these findings we should suspect the presence of GH secreting pituitary adenoma if high levels of serum GH and/or IGF-1 levels are detected at least twice, there is no GH suppression following a $75 \mathrm{~g}$ OGTT, or there is an abnormal GH response to TRH/LHRH. In these circumstances, we recommend that MET-PET fusion MRI should be performed for early detection of $\mathrm{GH}$ secreting pituitary adenoma.

\section{Conclusions}

Most patients with an early stage GH secreting adenoma have a microadenoma, which is often difficult to detect with superconductive MRI. Additionally, the results of loading tests are often inconclusive because there are marked fluctuations in plasma GH and IGF-1 levels. MET-PET fusion 3T-MRI method provides greater sensitivity for determining the location of and delineating GH secreting adenoma. In this report, we have emphasized the usefulness of MET-PET fusion MRI for the diagnosis of $\mathrm{GH}$ secreting adenoma if a GH producing tumor is suspected based on endocrine factors, which ultimately facilitates the early diagnosis and treatment of a GH secreting adenoma.

\section{References}

1. Ben-Shlomo A, Melmed S (2008) Acromegaly. Endocrinol Metab Clin North Am 37: 101-122.

2. Bonert V (2009) Diagnostic challenges in acromegaly: a case-based review. Best Pract Res Clin Endocrinol Metab 23: S23-30.

3. Chanson P, Salenave S, Kamenicky P, Cazabat L, Young J (2009) Pituitary tumours: acromegaly. Best Pract Res Clin Endocrinol Metab 23: 555-574.

4. Colao A, Ferone D, Marzullo P, Lombardi G (2004) Systemic complications of acromegaly: epidemiology, pathogenesis, and management. Endocr Rev 25 102-152.

5. Holdaway IM (2007) Excess mortality in acromegaly. Horm Res 68: 166-172.

6. Melmed S (2009) Acromegaly pathogenesis and treatment. J Clin Invest 119 3189-3202.

7. Baldys-Waligorska A, Krzentowska A, Golkowski F, Sokolowski G, Hubalewska- 
Citation: Satoh S, Ikeda H, Saginoya T, Watanabe K (2012) Composite MET-PET Fusion 3.0 Tesla MRI is a New Sensitive Method for the Detection of GH Secreting Adenomas with Abnormal GH Secretary Dynamics. J Metabolic Synd S2:005. doi:10.4172/2167-0943.S2-005

Page 5 of 5

Dydejczyk A (2010) The prevalence of benign and malignant neoplasms in acromegalic patients. Endokrynol Pol 61: 29-34.

8. Brooke AM, Drake WM (2007) Serum IGF-I levels in the diagnosis and monitoring of acromegaly. Pituitary 10: 173-179.

9. Colao A (2009) 5 Long-term acromegaly and associated cardiovascular complications: a case-based review. Best Pract Res Clin Endocrinol Metab 23: S31-S38.

10. Lombardi G, Galdiero M, Auriemma RS, Pivonello R, Colao A (2006) Acromegaly and the cardiovascular system. Neuroendocrinology 83: 211-217.

11. Melmed S, Colao A, Barkan A, Molitch M, Grossman AB, et al. (2009) Guidelines for acromegaly management: an update. J Clin Endocrinol Metab 94: 1509-1517.

12. Morvan D, Komajda M, Grimaldi A, Turpin G, Grosgogeat $Y$ (1991) Cardiac hypertrophy and function in asymptomatic acromegaly. Eur Heart J 12: 666672.

13. Vitale G, Pivonello R, Lombardi G, Colao A (2004) Cardiovascular complications in acromegaly. Minerva Endocrinol 29: 77-88.

14. Bergström M, Muhr C, Lundberg PO, Långström B (1991) PET as a tool in the clinical evaluation of pituitary adenomas. J Nucl Med 32: 610-615.

15. De Souza B, Brunetti A, Fulham MJ, Brooks RA, DeMichele D, et al. (1990) Pituitary microadenomas: a PET study. Radiology 177: 39-44.

16. Ikeda H, Abe T, Watanabe K (2010) Usefulness of composite methioninepositron emission tomography/3.0-tesla magnetic resonance imaging to detect the localization and extent of early-stage Cushing adenoma. J Neurosurg 112: 750-755.

17. Muhr C (2006) Positron emission tomography in acromegaly and other pituitary adenoma patients. Neuroendocrinology 83: 205-210.

18. Ikeda H (2011) Diagnosis of Cushing's disease (Chapter 12); In: Advances in Medicine and Biology, Vol23. Berhardt LV (ed.). Nova Science Publishers, Inc New York pp185-204
19. Castinetti F, Morange I, Dubois N, Albarel F, Conte-Devolx B, et al. (2009) Does first-line surgery still have its place in the treatment of acromegaly? Ann Endocrinol (Paris) 70: 107-112.

20. Laws ER, Vance ML, Thapar K (2000) Pituitary surgery for the management of acromegaly. Horm Res 53: 71-75.

21. Giustina A, Chanson P, Bronstein MD, Klibanski A, Lamberts S, et al. (2010) A consensus on criteria for cure of acromegaly. J Clin Endocrinol Metab 95 3141-3148.

22. Marro B, Zouaoui A, Sahel M, Crozat N, Gerber S, et al. (1997) MRI of pituitary adenomas in acromegaly. Neuroradiology 39: 394-399.

23. Pokrajac-Simeunovic A, Trainer PJ (2004) Pitfalls in the diagnosis of acromegaly. Horm Res 62: 74-78.

24. Freda PU (2009) Monitoring of acromegaly: what should be performed when GH and IGF-1 levels are discrepant? Clin Endocrinol (Oxf) 71: 166-170.

25. Doga M, Bonadonna S, Gola M, Nuzzo M, Giustina A (2005) Diagnostic and therapeutic consensus on acromegaly. J Endocrinol invest 28: 56-60.

26. Barkan AL (2004) Biochemical markers of acromegaly: GH vs. IGF-I. Growth Horm IGF Res 14: S97-S100.

27. Bonneville JF, Bonneville F, Cattin F (2005) Magnetic resonance imaging of pituitary adenomas. Eur Radiol 15: 543-548.

28. Newton DR, Dillon WP, Norman D, Newton TH, Wilson CB (1989) Gd-DTPAenhanced MR imaging of pituitary adenomas. AJNR Am J Neuroradiol 10: 949954

29. Soule SG, Jacobs HS (1996) The evaluation and management of subclinical pituitary disease. Postgrad Med J 72: 258-262.

30. Tabarin A, Laurent F, Catargi B, Olivier-Puel F, Lescene R, et al. (1998) Comparative evaluation of conventional and dynamic magnetic resonance imaging of the pituitary gland for the diagnosis of Cushing's disease. Clin Endocrinol (Oxf) 49: 293-300.
This article was originally published in a special issue, Pathophysiology and Treatment of Acromegaly handled by Editor(s). Dr. Hidetoshi lkeda, Southern Tohoku General Hospital, Japan 